\title{
COMBINED EPILEPSY/ADHD AND WORKING MEMORY
}

Researchers at University Children's Hospital Basel, and other centers in Switzerland, Norway, and Germany studied behavioral differences in working memory performance, response to methylphenidate, and functional brain organization in 17 boys with combined epilepsy/ADHD, 15 boys with developmental ADHD, and 15 healthy controls (aged 8-14 years). Boys with epilepsy-associated ADHD and those with ADHD without epilepsy both performed poorly on psychological tests with high cognitive load when compared to healthy controls. Methylphenidate improved cognitive performance almost to normal levels in both ADHD groups. Functional MRI showed similar reductions of activation in both ADHD groups, using working memory tasks. Boys with epilepsy/ADHD and ADHD without epilepsy recruited less cortical regions involved with working memory. ADHD with or without epilepsy show a common aberrant network of working memory. Following intake of methylphenidate, working memory was improved without alteration in the functional MRI. Seizures or administration of antiepileptic drugs in the epilepsy/ADHD group were not considered a cause of the working memory deficits. (Bechtel N, Kobel M, Penner I-K, et al. Attention-deficit/hyperactivity disorder in childhood epilepsy: a neuropsychological and functional imaging study. Epilepsia February 2012;53(2):325-333). (Respond: Dr Nina Bechtel, University Children's Hospital Basel, Spitalstrasse 33, 4056 Basel, Switzerland. E-mail: nina.bechtel@unibas.ch).

COMMENT. The authors conclude and confirm reports that ADHD with and without epilepsy represent epiphenomena of a common underlying functional and neurobehavioral pathophysiology, and that methylphenidate shows equivalent efficacy in both patient groups. Approximately 20\% children with epilepsy have ADHD (Gross-Tsur V, et al. J Pediatr 1997;130:670-674), and $-25 \%$ of children with ADHD have epileptiform, sleep-deprived EEGs (Millichap JJ, Stack CV, et al. J Child Neurol 2011;26(1):6-11).

A longitudinal follow-up study correlating clinical, neuropsychological, and EEG features with AED therapy reveals a temporal relation between subclinical epileptiform discharges, cognitive dysfunction, and effectiveness of AEDs on ADHD and EEG discharges (Laporte N, et al. Cognitive epilepsy: ADHD related to focal EEG discharges. Pediatr Neurol 2002;27:307-311). The authors' recommendation of AEDs in children with ADHD and abnormal EEG remains controversial and not generally accepted in practice. However, more recent studies confirm the reversal of transient cognitive impairment (TCI) with epileptiform EEG in children with ADHD without clinical seizures. (Schubert R. Pediatr Neurol 2005;32:1-10) (Mintz M, et al. J Child Neurol 2009;24(7):807-815). The doctrine or admonition, “Treat the patient's seizures and not the EEG," may require modification.

\section{RELATION OF COGNITIVE PROFICIENCY TO EPILEPSY FOCUS}

Investigators at Children's Memorial Hospital/Northwestern University School of Medicine Epilepsy Center, Chicago, and Kangbuk Samsung Hospital, Seoul, Korea examined the relationship of cognitive proficiency (CP) to general intellectual ability 